\title{
Arborescences
}

Revue d'études françaises

\section{La Seine de Mérimée et ses ressorts dramatiques}

\section{Janine Gallant}

Numéro 8, décembre 2018

La Seine littéraire au XIX ${ }^{\mathrm{e}}$ siècle

URI : https://id.erudit.org/iderudit/1055880ar

DOI : https://doi.org/10.7202/1055880ar

Aller au sommaire du numéro

Éditeur(s)

Département d'études françaises, Université de Toronto

ISSN

1925-5357 (numérique)

Découvrir la revue

Citer cet article

Gallant, J. (2018). La Seine de Mérimée et ses ressorts dramatiques.

Arborescences, (8), 6-16. https://doi.org/10.7202/1055880ar

\section{Résumé de l'article}

Si Mérimée, enclin à donner une toile de fond exotique à ses récits narratifs, accorde somme toute peu de place à la Seine, il lui confère en revanche un rôle fondamental dans son seul vrai roman, Chronique du règne de Charles IX. Tout en valant pour la pierre angulaire de l'oeuvre mériméenne, ce texte participe à l'invention romantique de la forme littéraire du roman historique. Cet article se penche sur l'utilisation que fait Mérimée de la Seine dans son écriture romanesque, telle qu'elle est conditionnée en partie par son rapport à l'Histoire. On se propose d'abord d'explorer l'écriture du roman historique telle que la conçoit Mérimée, puis d'analyser son traitement de la Seine en particulier. Le fleuve parisien prend des couleurs souvent dramatiques et est présenté comme une frontière à traverser, un axe ténébreux qui scinde la ville en des mondes distincts. 


\section{SOMMAIRE}

1 Nicolas Gauthier, Université de Waterloo

Sébastien Roldan, chercheur indépendant

Introduction

6 Janine Gallant, Université de Moncton

La Seine de Mérimée et ses ressorts dramatiques

17 Silvia Baroni, Université de Bologne

Au seuil de l'enfer: la Seine dans La Comédie humaine

33 Nicolas Gauthier, Université de Waterloo

La Seine du crime et les petits métiers parisiens

46 Sébastien Roldan, chercheur indépendant

Où est la Seine dans les Tableaux parisiens de Baudelaire?

64 Valérie Narayana, Université Mount Allison

Géocritique du fleuve dans les mémoires de la Commune de Louise Michel

80 Peter Vantine, Saint Michael's College

Au bord de la Seine avec les Goncourt

102 Lola Kheyar Stibler, Université Sorbonne Nouvelle

«Hydrargyre» de Maurice de Fleury ou l'imagination de la matière 


\title{
La Seine de Mérimée et ses ressorts dramatiques
}

\author{
Janine Gallant, Université de Moncton
}

\section{Résumé}

Si Mérimée, enclin à donner une toile de fond exotique à ses récits narratifs, accorde somme toute peu de place à la Seine, il lui confère en revanche un rôle fondamental dans son seul vrai roman, Chronique du règne de Charles IX. Tout en valant pour la pierre angulaire de l'œuvre mériméenne, ce texte participe à l'invention romantique de la forme littéraire du roman historique. Cet article se penche sur l'utilisation que fait Mérimée de la Seine dans son écriture romanesque, telle qu'elle est conditionnée en partie par son rapport à l'Histoire. On se propose d'abord d'explorer l'écriture du roman historique telle que la conçoit Mérimée, puis d'analyser son traitement de la Seine en particulier. Le fleuve parisien prend des couleurs souvent dramatiques et est présenté comme une frontière à traverser, un axe ténébreux qui scinde la ville en des mondes distincts.

Contrairement à la plupart de ses contemporains, Mérimée se montre a priori relativement discret par rapport à la Seine dans son écriture. Sa démarche artistique étant nourrie par ses nombreux voyages en dehors de la capitale parisienne, y compris ceux qu'il doit faire dans le cadre de ses fonctions d'Inspecteur des monuments historiques, la toile de fond de nombre de ses récits narratifs les plus célèbres est exotique (Carmen, Mateo Falcone) ou provinciale (La Vénus d'Ille). Comme l'explique Gisèle MathieuCastellani eu égard à son processus créatif en Corse, «Mérimée [...] est ainsi un voyageur qui cherche en pays exotique, dans une ville aussi étrange qu'étrangère, un dépaysement, c'est-à-dire une sortie de soi-même qui est aussi une rentrée en soi, dans cet autre moi, plus trouble, plus troublé, plus troublant» (2004: 114). Paris et le Nord de la France ne deviennent donc que parcimonieusement le cadre de l'univers littéraire mériméen. Les quelques nouvelles qui ont pour toile de fond la capitale française confinent le plus souvent les personnages dans des huis clos (salons, chambres, boudoirs), en dehors de quelques sorties dans des bois, ruelles et parcs, mais jamais véritablement près du fleuve.

Il est une œuvre qui fait exception à cette règle dans la carrière de Mérimée, et qui confère un véritable rôle à la Seine en la présentant, de manière dramatique, comme un seuil à franchir, un axe ténébreux qui scinde la ville en deux mondes distincts et opposés. Il s'agit du seul vrai roman de l'auteur, Chronique du règne de Charles $I X^{1}$. Ce texte est d'autant plus intéressant qu'il s'inscrit dans le mouvement de création du roman historique romantique. En effet, comme sujet à son roman, paru en 1829, mais rédigé en 1828, Mérimée choisit les guerres de Religion du Xvi ${ }^{\mathrm{e}}$ siècle, ce qui le place très clairement dans les premières tentatives d'exploration du genre. Dans le cadre de cet article, nous souhaitons donc envisager la manière très particulière dont Mérimée utilise l’image de la Seine dans

1. Désormais désigné sous le nom "Chronique», dans les références. Le roman paraît d'abord sous le titre de 1572 , Chronique du temps de Charles IX. Lors de sa première réédition en 1832, le titre devient 1572, Chronique du règne de Charles IX, "sans doute pour éviter un pléonasme entre chronique et temps» (1978: 1226) supposent Jean Mallion et Pierre Salomon, les éditeurs de l'édition de la Pléiade. Pierre Josserand, dans son édition du roman, est plus catégorique: "Mérimée corrige le pléonasme qui, bien qu'il sût le grec, lui avait d'abord échappé» (1969: 11). La date n'apparaît plus dans l'édition de Charpentier en 1842. 
son écriture romanesque, dictée en partie par son rapport à l'Histoire. Pour ce faire, nous commencerons par considérer la façon générale dont Mérimée conçoit l'écriture du roman historique durant cette période où les écrivains français cherchent à lui ouvrir de nouvelles avenues. L'auteur de la Chronique du règne de Charles $I X$ développe une approche personnelle et encore assez exploratoire du genre, où il importe notamment de véritablement transporter le lecteur dans une période historique sur laquelle l'auteur s'est largement documenté. Cette démarche forge le traitement qu'il réserve à la Seine dans son roman et que nous analyserons, dans un second temps, à travers les figures qui lui sont associées, tels les ponts qui la surplombent et les bateaux qui la traversent.

\section{Mérimée et le roman historique}

Au moment où Mérimée entreprend l'écriture de son premier et unique roman, il participe à l'expérience nouvelle du roman historique romantique. Rappelons en effet que, comme l'a bien montré Georges Lukacs, "[l]e roman historique est né au début du XIX ${ }^{\mathrm{e}}$ siècle, à peu près à l'époque de la chute de Napoléon (Waverley de Walter Scott a paru en 1814)» (2000: 17). Walter Scott introduit donc une nouvelle forme romanesque qui a tôt fait de fasciner les écrivains français. Dans un article qui lui est consacré, paru dans La Muse française en 1823, Hugo pose bien les enjeux et intérêts de ce nouveau genre littéraire: "Walter Scott [...] a su puiser aux sources de la nature et de la vérité un genre inconnu, qui est nouveau parce qu'il se fait aussi ancien qu'il le veut» (1907: 27). Ainsi, une véritable "vogue de Walter Scott imprègne la production d'innombrables écrivains» (Bernard 2008: 19). Parallèlement, l'intérêt grandissant de la société française pour l'Histoire et le développement de cette dernière comme discipline contribuent à l'essor du roman historique dans la première moitié du XIX ${ }^{\mathrm{e}}$ siècle. Vigny, dans sa préface ${ }^{2}$ à Cinq-Mars, roman paru en 1826 et souvent considéré comme le premier véritable roman historique français, signale que "[d]ans ces dernières années (et c'est peut-être une suite de nos mouvements politiques), l'Art s'est empreint d'histoire plus fortement que jamais» (Cinq-Mars: 21). C'est donc dans ce climat général que Mérimée compose son roman, "deuxième essai marquant du nouveau roman historique français ", selon Sarah Mombert (2000: 128).

Il faut dire que l'attrait de l'Histoire n'est pas nouveau pour Mérimée, dont la carrière avait commencé avec des textes de théâtre, en bonne partie exotiques, reflétant son penchant pour l'étranger, mais comportant aussi, le plus souvent, une dimension historique. Ainsi, au moment où il entreprend la Chronique du règne de Charles $I X$, il a déjà une certaine expérience de la littérature historique qu'il poursuivra d'ailleurs dans la suite de sa carrière. Xavier Bourdenet, dans son analyse des Faux Démétrius (1852), perçoit «une écriture de l'histoire qui, si elle se donne comme but la vérité et se définit donc contre le faux, appelle néanmoins un prolongement dans la fiction» (2009: 35-36). Mérimée consacre ainsi une bonne partie de sa carrière d'écrivain à tenter d'insuffler la vérité historique à l'intérieur de la fiction, à tel point que l'étiquette d'«historien» lui est parfois attribuée, y compris par lui-même, comme lorsqu'il écrit soudain dans sa nouvelle Le Vase étrusque (1830): "Mon devoir d'historien m’oblige à déclarer qu'une nuit du mois de juillet [...]»(1978: 513), alors qu'il conte une histoire fictive. Avec la Chronique du règne de Charles IX, il canalise cet intérêt pour l'Histoire dans le genre du roman qu'il n'a pas encore exploré et auquel il ne reviendra plus par la suite puisque, comme on le sait, il privilégiera finalement la forme brève.

2. Rédigée pour la réédition du roman en 1829. 
Le roman historique est un genre complexe et protéiforme, pour lequel il est encore aujourd'hui difficile de trouver une définition satisfaisante, mais dont l'une des particularités est de réunir deux éléments souvent perçus comme contraires. Isabelle Durand-Le Guern rappelle qu'on "peut de fait constater une sorte de dualité irréductible du roman historique: d'un côté, la fiction et l'aventure, de l'autre, l'information et l'histoire» (2008: 93). C'est tout le problème de la "soudure» de ces deux éléments, celle qui lie «l'histoire au roman, le réel à la fiction", pour reprendre la formule de Jean Molino (1975: 195). Le souci de cette «soudure» habite certainement Mérimée, dont la nature de documentariste se révèle à l'approche du roman. Jean Mallion et Pierre Salomon, après l'analyse comparative des placards publicitaires des deux premiers romans historiques français (celui de Mérimée et celui de Vigny), concluent:

On le voit, deux conceptions du roman historique s'opposent. Vigny dédaigne comme trop «vulgaires» les recherches historiques et n'est préoccupé que de "vues philosophiques et morales». Mérimée, au contraire, plus modestement, ne vise qu’à reconstituer une époque à l'aide de «documents originaux» $(1978: 1221)$.

Ces deux auteurs affirment même que «Mérimée n’est pas un conteur spontané. Son imagination a besoin d'un tremplin: document, détail vécu» (1978: 1216). L'auteur de la Chronique du règne de Charles IX se trouve donc dans une situation particulière à deux égards: d'une part, il expérimente dans le cadre de sa propre création littéraire, en composant pour la première fois avec le genre romanesque; d'autre part, sa pratique de l'œuvre historique s'inscrit à contre-courant des vues de certains de ses contemporains.

En effet, Mérimée cherche à transporter "véritablement» son lecteur dans une autre période historique. On le sent dès les premières lignes du roman. Comme le dit Jean-Louis Cabanès, pour la démarche générale de Mérimée, «le lecteur doit avoir le sentiment que l'écrivain a reconfiguré les mœurs comme s'il avait pu les saisir dans leur état natif» (2012: 11). Hippolyte Taine, dans sa perspective privilégiée d'historien, lui accordait d'ailleurs le don de "faire revivre des débris morts» (1874: XVII). À cet égard, l'incipit du roman est révélateur de la façon dont Mérimée procède pour imposer un retour dans le temps. Dès les premiers paragraphes, le narrateur déplace en effet habilement son lecteur contemporain vers la période antérieure visée par le roman:

Non loin d'Étampes, en allant du côté de Paris, on voit encore un grand bâtiment carré, avec des fenêtres en ogive, ornées de quelques sculptures grossières. Au-dessus de la porte est une niche qui contenait autrefois une madone de pierre; mais dans la révolution elle eut le sort de bien des saints et des saintes, et fut brisée en cérémonie par le président du club révolutionnaire de Larcy [...]. Depuis on a remis à sa place une autre vierge, qui n'est que de plâtre à la vérité, mais qui, au moyen de quelques lambeaux de soie et de quelques grains de verre, représente encore assez bien, et donne un air respectable au cabaret de Claude Giraut.

Il y a plus de deux siècles, c'est-à-dire en 1572, ce bâtiment était destiné, comme à présent, à recevoir les voyageurs altérés; mais il avait alors une tout autre apparence. Les murs étaient couverts d'inscriptions [...] (Chronique: 47).

Le "encore» de la première phrase du roman signale un déjà-là, la connaissance de l'existence passée de ce lieu et force d'emblée le lecteur à considérer l'historicité du décor mis en place. Puis s'opère une sorte de télescopage de périodes historiques, alors qu'on assiste à une série de va-et-vient entre diverses époques, du présent, au général "autrefois », à l'époque de la Révolution, à 1572, et ce, sans compter les 
alternances de temps verbaux entre le présent et le passé. L'incipit du roman permet donc de conduire le lecteur de son présent contemporain à la fin du XvI ${ }^{\mathrm{e}}$ siècle, en soulignant partout au passage les marques laissées par l'Histoire. L'auteur s'assure ainsi de ne pas rompre brutalement et immédiatement avec la période moderne, amenant son lecteur presque progressivement au passé. D’ailleurs, Mérimée maintiendra régulièrement ce lien avec le lecteur contemporain, en prenant la peine de lui rappeler qu'il a été transporté dans un autre Paris que celui qu'il connaît. Pour faciliter la transition vers le $\mathrm{XVI}^{\mathrm{e}}$ siècle, par exemple, Mérimée glisse à l'occasion des notes explicatives. Ainsi, alors qu'il est question du «Pré-aux-Clercs» dans une conversation entre personnages, on lira la note suivante de Mérimée lui-même: «lieu classique alors pour les duels, le Pré-aux-Clercs s'étendait en face du Louvre, sur le terrain compris entre la rue des Petits-Augustins et la rue du Bac» (Chronique: 92). Ou encore, le narrateur signale au passage, dans le corps du texte: "près du lieu où l'on bâtissait le château des Tuileries, commencé en 1564 » (93); «dans presque toutes les maisons de Paris, il y avait à la porte d'entrée une petite ouverture carrée, avec un grillage de fer très serré» (271). Mais ces notes explicatives sont utilisées avec parcimonie; il ne s'agit en rien de donner un cours sur la topographie parisienne du Xvi ${ }^{\mathrm{e}}$ siècle, mais plutôt de s'assurer que le lecteur s'y retrouve, en lui fournissant quelques repères au passage, sans toutefois briser le flux du récit. De surcroît, le narrateur fera aussi des remarques à son lecteur pour lui rappeler le caractère historique du roman et bien lui faire ressentir l'époque passée, par exemple: «car les rues de Paris après huit heures du soir, étaient alors plus dangereuses que la route de Séville à Grenade ne l'est encore aujourd'hui» (119).

\section{La Seine du xvl ${ }^{e}$ siècle}

Mérimée restitue si bien l'époque où se déroule son roman que Jean-Louis Cabanès identifie dans la Chronique du règne de Charles IX une "couleur seiziémiste de l'énoncé» (2012: 11). En ce sens, l'évocation de la Seine représente un défi particulier pour le romancier, soit de ramener le lecteur à la Seine ancienne. Dès lors, tout se passe comme si Mérimée ne cherche pas à ouvrir la voie à une représentation mélancolique du fleuve parisien, celle qui sera pourtant mise en avant par d'autres auteurs quelques années plus tard et qui deviendra une caractéristique de la représentation littéraire de Paris par les romantiques. Comme on le sait, ces derniers attribuent à l'eau - cette «insoumise par excellence [qui] peut modifier son allure, mais [...] garde son éclat et sa voix", comme le dit George Sand (La Rêverie à Paris: 26) - une portée émotive, mais aussi un aspect mélancolique, à l'instar du fleuve de Lamartine, qui, dans "L'isolement", "gronde», "serpente, et s'enfonce en un lointain obscur» (Méditations poétiques: 3). La Seine fera donc tout naturellement partie de ces fleuves qui invitent à la contemplation. Cette vision héritée du romantisme trouve d'ailleurs encore ses échos aujourd'hui lorsqu'est évoqué le rôle de la Seine dans la vie du Parisien:

Qui n'a un jour fait un arrêt au milieu d'un pont et ne s'est penché pour regarder passer le fleuve? Être au milieu de l'eau, pouvoir scruter son mystère, imaginer ce qu'elle recèle, examiner sans fin le jeu des vagues, des remous et des tourbillons. [...] Un pont jeté sur la rivière permet ce moment de pause. [...] C'est un poste d'observation privilégié (Bernard 2009: 58).

Ainsi, on croisera souvent chez les auteurs de la première moitié du XIX ${ }^{\mathrm{e}}$ siècle des personnages qui contemplent la Seine, réfléchissent à ses bords, se plongent en des "rêveries». Même les rentiers de Balzac ne peuvent s'empêcher de poser régulièrement leur regard sur la Seine: 
La Seine, cette belle reine, n'aurait plus ses courtisans: le Rentier ne va-t-il pas la voir quand elle charrie, quand elle est prise en entier, quand elle arrive au-dessus de l'étiage inscrit au pont Royal, quand elle est à l'état de ruisseau, perdue dans les sables du bras de l'Hôtel-Dieu? En toute saison, le Rentier a des motifs pour aller contempler la Seine (Balzac, Monographie du Rentier: 11).

Sans oublier l'attrait plus tragique de la Seine quand les personnages de romans romantiques songent à se jeter dans le fleuve, dont l'exemple le plus connu est probablement le Raphaël de La Peau de chagrin de Balzac:

Il s'achemina vers le pont Royal [...] en se serrant contre le parapet du pont [...] il regarda l'eau d'un air sinistre.

- Mauvais temps pour se noyer lui dit en riant une vieille femme vêtue de haillons. Est-elle sale et froide, la Seine! (Balzac, La Peau de chagrin: 71).

Si Mérimée n'est pas nécessairement réfractaire à cette vision de la Seine, l'enracinement de son intrigue dans l'univers du Xvi ${ }^{\mathrm{e}}$ siècle et dans le cadre d'un roman historique le pousse à restituer avec le plus de justesse possible le rapport qu'entretenaient les Parisiens avec leur rivière à cette époque. En examinant de plus près les passages où il est question de la Seine dans la Chronique du règne de Charles IX, on constate que Mérimée semble vouloir surtout faire ressortir le seuil qu'elle représente, à la manière d'un axe qui délimiterait des mondes distincts et qui demanderait à être franchi. L'image des eaux qu'il faut traverser (soit par un pont, soit en bateau) est mise en avant et culmine dans une scène déterminante et saisissante du roman, laquelle prend place juste avant le massacre de la Saint-Barthélemy.

\section{Le pont}

Qu'il nous soit permis ici de tracer les grandes lignes de l'intrigue de ce roman historique à caractère encore assez expérimental ${ }^{3}$ afin de mieux contextualiser l'analyse qui va suivre. Comme l'indique l'incipit cité plus haut, l'histoire débute dans une auberge "[n] on loin d'Étampes» en 1572. Le lecteur y fait la connaissance de Bernard de Mergy, jeune huguenot en route vers Paris et personnage principal du roman, le plus souvent simplement désigné "Mergy» par le narrateur. Arrivé dans la capitale, il y croise par hasard son frère George ${ }^{4}$, qu'il a perdu de vue depuis la conversion de ce dernier au catholicisme. George initie alors son jeune frère à la Cour du roi, où il a ses entrées. Lors d'une soirée mondaine, Mergy tombe sous le charme de Diane de Turgis, noble catholique. S’ensuit une tentative de séduction remplie de péripéties, dont un duel gagné par Bernard mais qui le laisse blessé. Le massacre de la Saint-Barthélemy frappe aux deux tiers du roman. Bernard y survivra, protégé par Diane, qui cherche à le convertir au catholicisme. Le roman se clôt sur un long épisode consacré au siège de La Rochelle, où, à leur insu, les deux frères combattent dans des camps ennemis.

Le héros protestant fait donc son entrée à Paris, «le soir [...], peu de temps avant la fermeture des portes» (Chronique: 80 ), dès la fin du deuxième chapitre du roman. La première activité parisienne

3. «Nous avons donc ici une très sérieuse tentative de continuer le roman historique sur la base de l'exploration», affirme Lukacs (2000: 85). Comme le résume bien Aude Déruelle, la Chronique du règne de Charles IX peut être en partie conçue comme «la présentation de nombreuses pistes (ou fausses pistes) offertes à un lecteur en attente d'un sens à saisir» (2010: 54).

4. Dans le compte rendu qu'il rédige pour Le Globe, Charles Magnin considère que George est le véritable héros du roman, «celui qu'au fond l'on aime et l'on estime le plus» (1829: 342). 
de Mergy se résume ainsi : «il sortit de son hôtellerie pour aller chez un orfèvre du pont Saint-Michel » (Chronique: 82). Il s'agit d'un épisode clé du roman, car c'est sur ce pont que Bernard va croiser par hasard son frère George, qu'il n'a pas vu «depuis plus de sept ans». Cette rencontre, Bernard en a anticipé l'éventualité, non sans s’interroger sur la conduite qu’il devrait adopter le cas échéant, puisque la conversion au catholicisme de George «l'avait presque entièrement séparé de sa famille, pour laquelle il n'était guère plus qu'un étranger" (Chronique: 81). L'épisode permet déjà d'appréhender le pont qui traverse la Seine comme un lieu de confrontation entre deux mondes (ici, les deux frères du roman, appartenant à des religions différentes, celles qui font l'objet des tensions de l'époque). Ce moment passé sur le pont est aussi l'occasion d'une autre rencontre pour le héros, à savoir celle de Béville, qui fait partie du groupe d'amis catholiques accompagnant George. Cet événement n'est pas sans importance, car le personnage de Béville sera un élément central d'autres épisodes dont nous parlerons plus loin.

S’avère également révélateur le choix des informations fournies par le narrateur au sujet de ce pont Saint-Michel : «[à] l'entrée du pont», un groupe de personnes, «jeunes gens vêtus avec beaucoup d'élégance», "barraient presque entièrement le passage étroit que laissaient sur le pont la multitude de boutiques et d'échoppes qui s'élevaient comme deux murs parallèles» (Chronique: 82). L'accès au passage de la Seine est donc difficile et la traversée peu agréable pour le héros. Qui plus est, ces «murs» de boutiques "dérobaient complètement la vue de la rivière aux passants» (Chronique: 82). S’il est vrai, comme le constate l'écrivain Nicolas d'Estienne d'Orves, que «[1]'histoire des ponts de Paris est celle de leur édification et de leur démolition, de leurs reconstitutions et de leurs déplacements: une épopée toujours changeante, toujours mouvante»(2015: 492), il serait opportun de rappeler ici que c'est seulement en 1786 qu'un édit du Conseil du Roi amorce le processus de destruction des maisons qui restaient encore sur les ponts de Paris; celles du pont Saint-Michel, les dernières, disparaîtront en 1808 (Carbonnier 1998). Le retour à une Seine du Xvi ${ }^{\mathrm{e}}$ siècle, où les ponts sont encore chargés de bâtiments, exclut donc la possibilité d'une contemplation romantique des eaux, familière au lecteur contemporain de Mérimée. Par ailleurs, ce détail supplémentaire renforce l'image d'une forme d'inaccessibilité de la Seine. Ainsi, dès ce début de roman, plus que toutes les images contemplatives qui pourraient être associées à la Seine, c'est sa traversée qui est mise en relief.

Comme pour accentuer encore davantage l'importance de cet épisode, c'est précisément sur ce pont étroit et rempli d'embûches que s'amorce véritablement la découverte de Paris par le héros, son frère lui lançant: «ainsi viens avec nous. Tu vas faire connaissance avec la vie de Paris» (Chronique: 85). Ce qui est ainsi évoqué, c'est un apparent rite initiatique, lequel suppose toujours un passage à franchir.

Par contraste, le pont Saint-Michel de la fin du Xvi ${ }^{\mathrm{e}}$ siècle sera évoqué bien différemment dans l'univers romanesque de Dumas, par exemple lorsque l'auteur des Trois Mousquetaires proposera son propre roman historique basé sur les guerres de Religion: La Reine Margot (en feuilleton à partir de 1844). Il s'agit sans doute de la représentation romanesque la plus connue de la période historique entourant le massacre de la Saint-Barthélemy durant la première moitié du XIX ${ }^{\mathrm{e}}$ siècle français ${ }^{5}$. En effet, lorsqu'il y est également question du pont Saint-Michel, les explications historiques d'usage sont déjà bien plus étoffées que celles que Mérimée peut fournir:

Le pont Saint-Michel avait été bâti en pierres en 1373: malgré son apparente solidité, un débordement de la Seine le renversa en partie le 31 janvier 1408; en 1416, il avait été reconstruit en bois;

5. Cette période historique inspire bon nombre d'œuvres au XIX ${ }^{\mathrm{e}}$ siècle, y compris un opéra d'Hérold et du librettiste Eugène de Planard, directement inspiré du roman de Mérimée: Le Pré-aux-Clercs (1832). Voir à ce sujet notamment l'article d'Olivier Bara dans les Cahiers de Mérimée (Bara 2015). 
mais pendant la nuit du 16 décembre 1547 il avait été emporté de nouveau; vers 1550, c'est-à-dire vingt-deux ans avant l'époque où nous sommes arrivés, on le reconstruisit en bois, et, quoiqu'on eût déjà eu besoin de le réparer, il passait pour assez solide (Dumas, La Reine Margot: 294).

Le narrateur dumasien s'attarde sur ce pont surtout pour concentrer l'attention du lecteur sur un aspect en particulier: la maison de «M. René le parfumeur», qui deviendra l'un des lieux de l'action: «on remarquait une maison à panneaux de bois sur laquelle un large toit s'abaissait comme la paupière d'un œil immense [...] avec cette inscription: René, Florentin, parfumeur» (2956). Dumas décrit, avec force détails, l'une des maisons qui ne sont mentionnées chez Mérimée que pour souligner le fait qu'elles obstruent la vue et rendent toute contemplation de la Seine impossible.

\section{La traversée en bateau}

La Seine est évoquée à nouveau dans la Chronique une dizaine de pages après la première traversée par les deux frères. Il s'agit d'une mention furtive qui permettra toutefois d'inscrire dans le texte le présage d'un événement plus important à venir. Une querelle dans un restaurant entre le chevalier de Vinci et Vaudreuil tourne au duel. Au cours de celui-ci, le chevalier est blessé: «bientôt parurent un moine et un chirurgien, qui se disputèrent quelque temps le blessé. Le chirurgien cependant eut la préférence, et, ayant fait transporter son patient au bord de la Seine, il le conduisit dans un bateau jusqu'à son logement» (Chronique: 97). La Seine à franchir, les moyens dont on dispose pour ce faire (cette fois le bateau), autant d'éléments qui évoquent toujours l'image d'un fleuve-obstacle qu'il faut passer pour accéder à un autre univers.

Cette idée sera renforcée au moment où Mérimée montre son héros à son tour sommé d'entrer en duel - non sans avoir manqué de signaler à son lecteur qu'il s'agit d'une pratique alors courante: "[c]ette querelle et le combat irrégulier qui l'avait suivie n'avaient rien d'extraordinaire à cette époque" (Chronique: 99). Cette fois, un rendez-vous a été fixé et il faut se rendre au Pré-aux-Clercs. Rappelons que, comme le précise Mérimée dans l'une de ses notes explicatives, le Pré-aux-clercs se situait sur la rive gauche, "entre la rue des Petits-Augustins et la rue du Bac» (92). Les Mergy logeant sur la rive droite, une première traversée de la Seine va donc s'imposer pour rejoindre le lieu du duel avant même de pouvoir espérer en revenir. À cette fin, ils auront recours aux services d'un passeur, ce qui est indiqué dans le roman à l'aide d'une formule quelque peu laconique: «Ils entrèrent dans un bateau et traversèrent la Seine.» (166) La narration souligne aussi que Comminges et son second, Béville, les autres parties prenantes du duel, atteignent également l'endroit désigné à l'aide d'un bac: "Au moment où ils abordèrent, ils aperçurent un bateau chargé de deux hommes et traversant la rivière quelque cent pieds plus bas. - Voici nos gens [...]» (166-167). Puis, par le biais du personnage de Comminges, le narrateur rappelle que ce "passage» ne se fait pas sans difficulté. Lorsque George tente de désamorcer la querelle, et donc le duel, au bénéfice de son jeune frère Bernard, la réplique de Comminges est sans appel et met en relief le "voyage» qui a été effectué pour venir au duel: «me croyez-vous homme à

6. Il est fait allusion à cette maison du pont Saint-Michel ailleurs dans le roman de Dumas: «- C'est prévu. Il dira qu'il vient de la part de M. René le parfumeur. - Ce Florentin qui demeure sur le pont Saint-Michel? - Justement» (La Reine Margot: 277) ; “C'était la première fois que la reine et l'astrologue se revoyaient depuis la visite que la reine lui avait faite à sa boutique du pont Saint-Michel» (521); «- Maître René! cria-t-il, maître René le Florentin! Qu’on coure au pont Saint-Michel, et qu'on me l'amène» (655). 
quitter le lit de ma maîtresse de si bonne heure... à traverser la Seine, le tout pour faire des excuses à un morveux?» (167)

Après le duel, Bernard et George, rejoints par Béville, sont contraints de reprendre le bateau pour retraverser la Seine. Le batelier reçoit alors l'ordre de «ram[er] comme [s'il] voulai[t] gagner une pistole»(Chronique: 173), car la Seine doit être franchie le plus rapidement possible. En effet, Béville s'exclame: "voici les gens avec des hallebardes qui s'avancent vers nous. Ce sont Messieurs les sergents qui s'en viennent de leur tour de Nesle, et nous ne voulons rien avoir à démêler avec eux» (173). Cette scène effrénée clôt un chapitre pour laisser place à l'ouverture du chapitre suivant, qui réactualise immédiatement la menace que fuient les personnages en traversant la Seine. Le douzième chapitre du roman débute en effet avec la phrase suivante: "Ces hommes armés de hallebardes étaient des soldats du guet, dont une troupe se tenait toujours dans le voisinage du Pré-aux-Clercs pour être à portée de s'entremettre dans les querelles qui se vidaient d'ordinaire sur ce terrain classique des duels» (175). Ce n'est que deux pages plus loin, après une longue discussion à bord du bateau, qu' «ils abord[ent] à la rive droite» (177). Mérimée fait ainsi ressentir au lecteur la durée nécessaire pour "traverser» la Seine (rappelons qu'en plus le batelier avait reçu l'ordre de ramer très vite), sans pour autant que les personnages prennent le temps de tourner le regard vers le fleuve sur lequel ils voguent: les deux pages qui séparent le départ en bateau du Pré-aux-Clercs et l'arrivée sur la rive droite ne sont occupées que par du dialogue brut et continu; aucune indication du narrateur sur l'apparence de la Seine ou sur une quelconque interaction entre les personnages et le fleuve. C'est une fois de plus son passage, complexe et ardu, qui est mis en relief.

En outre, ce long voyage est nécessaire pour passer d'un monde à un autre, ce qui est souligné par divers procédés. En effet, le narrateur accentue les contrastes entre les deux univers que sépare la Seine, en commençant par la description de la maison de George, que les deux frères doivent quitter pour se rendre au duel. Il s'agit d'un lieu enchanteur, presque féérique où «tous les deux s'assirent devant une table couverte de gâteaux de plusieurs sortes, accompagnés d'un grand pot d'argent rempli de vin " (Chronique: 166), quelques heures avant de se prévaloir des services d'un batelier pour se rendre au Pré-aux-Clercs. Le logement du capitaine George avait déjà été présenté au lecteur sous des aspects chatoyants à travers la focalisation de Mergy: «le logement du capitaine était meublé avec élégance. Des rideaux de soie à fleurs et des tapis de couleurs brillantes attirèrent d'abord les yeux de Mergy, accoutumé à plus de simplicité» (99). Dans ce lieu presque magique apparaît aussi "un petit laquais apport[ant] des confitures, des dragées et du vin blanc» (100), reflétant une fois de plus l'abondance qui règne en ces lieux. Dès lors, un violent contraste s'établira avec le décor rejoint par les personnages de l'autre côté de la Seine: on s’y salue "gravement», on y fait "une grimace négative» (167) et l'on remplit les discussions «d'injures» (168). Sans compter les sombres détails de l'action abritée par ce lieu, le duel où Comminges perd la vie, et les répercussions psychologiques éprouvées par Bernard: «Mergy se mit à trembler de tous ses membres, et de grosses larmes coulaient une à une sur ses joues" (172), car il s'agit de son premier combat ${ }^{7}$. On trouve donc associées au passage de la Seine des résonances du rite initiatique de Mergy qui avait été entamé sur le pont Saint-Michel, au début du roman. Ainsi s'accuse l'image d'une Seine ménageant un passage entre deux mondes, un passage essentiel, vital, mais difficile à pratiquer.

7. Tout ceci participe d'ailleurs des tensions générales présentes dans le roman. Comme le souligne Claudie Bernard, «[l]a tension inhérente au roman historique comme genre semble se répercuter à tous les niveaux de l'œuvre de Mérimée dont la partie romanesque a pour moteur les dissensions fraternelles et la partie historique d'âpres luttes religieuses" (1990: 109). 


\section{La Seine et le massacre}

Or, cette image de la Seine, qui s'inscrit par accumulation dans le roman, va permettre de donner une densité dramatique sans pareille à un épisode clé où le nom du fleuve parisien sera prononcé sans cesse, tel un mantra. Il s'agit du moment où, aux trois quarts du roman, on s'apprête à basculer vers le massacre de la Saint-Barthélemy, massacre que le lecteur a anticipé avec effroi. Rappelons en effet que c'est l'un des ressorts du roman historique que de compter sur la connaissance préalable du lecteur de certains événements de l'Histoire qui vont s'inscrire dans la trame narrative. Le narrateur annonce justement qu'il s'agit du «soir du 24 août» (Chronique: 243 ) et le lecteur a donc conscience que le récit est arrivé à la date fatidique. Comme il le fait régulièrement depuis déjà un moment, Bernard (huguenot, rappelons-le) sort ce soir-là pour se rendre chez l'élue de son cœur, Diane de Turgis, qui loge également sur la rive droite, rue des Assis ${ }^{8}$. Signalons au passage que Mérimée tire encore profit ici des contrastes violents auxquels il a habitué son lecteur, en décrivant cette soirée du point de vue de Bernard: «[1]a nuit était belle [...] c'était une nuit faite pour l'amour» (260). Bernard, ne soupçonnant évidemment rien des événements à venir que le lecteur connaît, remarque pourtant des choses étranges: beaucoup d'agitation dans la rue d'habitude peu fréquentée à cette heure, dont "des crocheteurs portant sur leurs épaules des fardeaux d'une forme étrange» (256). Puis, il se trouve croiser Béville. Ce dernier, catholique haut placé, est bien évidemment dans la confidence de l'événement terrible qui se prépare (il est d'ailleurs muni d'une cotte de mailles et d'armes dissimulées sous son manteau). S'abstenant de révéler à Mergy les projets réels de son camp, il ne peut toutefois s'empêcher de fournir un avertissement voilé à son interlocuteur, en lui suggérant d'abandonner son projet de rendez-vous galant et de traverser plutôt la Seine, avant minuit. Cet avertissement apparaît dès lors sous forme de sombres échos répétés au cours de la conversation: "passez la Seine ce soir même», "[p]assez la Seine, vous dis-je», "allez passer votre fureur de l'autre côté de la Seine», «[p] assez la Seine, mon brave; c'est mon dernier mot» (259-260). Lorsque Bernard demande à Béville de clarifier sa pensée, ce dernier lance une réplique finale: "Mon cher, je ne devrais pas peut-être vous parler si clairement; mais passez l'eau avant qu'il soit minuit; et adieu» (260). Mérimée emploie l'italique, comme pour faire culminer cet avertissement, après la répétition appuyée des mots "passez» et "Seine». Cette dernière revêt toujours les couleurs d'un axe central qu'il faut franchir pour passer d'un monde à l'autre, ici pour passer de la rive droite à la rive gauche, où l'on peut "plus facilement s'échapper de la ville et gagner les provinces du Midi» (278).

La mise en garde de Béville se présente au lecteur comme une sorte de dernière grâce possible pour le héros: fuir le danger en traversant les eaux parisiennes. Hélas, Bernard ne comprend pas l'avertissement (il ne peut évidemment pas concevoir la barbarie qui se prépare) et une sorte de décompte infernal s'enclenche, alors que le narrateur rend tangible le temps qui s'écoule: "En ce moment l'horloge sonna onze heures et demie» (Chronique: 263); "le son de cette horloge est affreux!» (263); «-Dans moins d'une heure, on va couper les sept têtes du dragon de l'hérésie. Les épées sont aiguisées et les fidèles sont prêts» (267); "tu as encore un quart d'heure pour te repentir» (267); "au bout de peu de minutes» (267); «le massacre est commencé! S’écria la comtesse en portant les mains à sa tête avec effroi » (268).

Le franchissement de la Seine, déjà présenté comme épineux, comme nous l'avons vu, est ce soirlà rendu impossible, comme l'explicite le texte à plusieurs reprises: «[p]ar une prévoyance infernale, la

8. Comme le signale Pierre Josserand dans les notes de son édition critique du roman: «[p]lus souvent désignée sous le nom de la rue des Arcis, la rue des Assis, actuellement partie de la rue Saint-Martin, joignait la rue Saint-Jacques-dela-Boucherie à la rue des Lombards» (Chronique: 362). 
plupart des bateaux qui d'ordinaire étaient amarrés le long du Louvre avaient été conduits sur l'autre rive» (274) tandis que «[d]es corps de garde nombreux occupaient les ponts» (277). Ainsi, les deux modes de traversée évoqués plus tôt dans le roman s'éclipsent soudainement. Dès lors, il n'est plus possible de "passer les eaux», comme l'avait suggéré Béville. Le passage de la Seine bloqué, cette dernière n'est plus que le symbole brutal de l'événement historique de 1572 en s'imprégnant du sang des victimes, symbole convenu dans les sources consultées par Mérimée, qui cite une formule empruntée à D’Aubigné: «là, selon l'expression énergique d'un écrivain contemporain [D’Aubigné, Histoire universelle, précise Mérimée en note], le sang courait de tous côtés cherchant la rivière» (273). L'auteur inscrit encore l'écho de cette image, vers la toute fin du roman, mais en la joignant cette fois à la notion du "passage» qu'il a tellement développée auparavant: "Ah! monsieur, si vous aviez vu les massacres du 24 août! si vous aviez passé la Seine quand elle était rouge» (312).

En somme, lorsque Mérimée rédige son premier et seul véritable roman, paru en 1829, il décide, lui qui est d'ordinaire plus attaché aux décors exotiques, d'adopter Paris comme toile de fond. Ce faisant, il est l'un des premiers auteurs du romantisme à développer une représentation littéraire de la Seine. Toutefois, il n'introduit pas, comme on aurait pu le croire au départ, le motif d'une contemplation émotive des eaux du fleuve parisien qui sera adopté par bon nombre d'auteurs romantiques quelques années plus tard. Au contraire, la nature historique de son œuvre et son approche personnelle et singulière de ce genre littéraire vont lui dicter un autre recours à la Seine. Par le biais d'une série de processus narratifs, le fleuve de la capitale est présenté sous l'angle d'un axe qui divise les mondes et qu'il faut franchir, quand bien même ce passage s'avère le plus souvent ardu, avant de devenir impossible au moment du massacre de la Saint-Barthélemy. Frontière devenant infranchissable, la Seine est ainsi chargée d'une densité dramatique remarquable qui lui donne un rôle tout à fait particulier, et absolument crucial, dans le seul roman mériméen.

\section{Références bibliographiques}

Balzac, H. 1995. La Peau de chagrin. Édition préparée et annotée par J. Martineau. Paris: Librairie générale française. [1831].

Balzac, H. 2012. Monographie du Rentier. Le Thoronet: Éditions de la Première Heure. [1841].

Bara, O. 2015. «Deux opéras pour un roman. Chronique du règne de Charles IX, Le Pré-aux-Clercs, Les Huguenots». Cahiers Mérimée 7: 61-75.

Bernard, C. 1990. "Roman historique et double jeu romanesque: la "Chronique du règne de Charles IX" de Mérimée». Romantisme 69: 97-111.

Bernard, C. 2008. "Si l'histoire m'était contée...». Dans Problèmes du roman historique, sous la direction d'A. Déruelle et A. Tassel. Paris: L'Harmattan: 15-25.

Bernard, J. 2009. Un fleuve et ses ponts - La Seine de Paris à Bougival. Saint-Cyr-sur-Loire: Éditions Alan Sutton.

Bourdenet, X. 2009. "Faux et usage de faux: l'histoire et son écriture dans Les Faux Démétrius de Mérimée». Cahiers Mérimée 1:33-55.

Cabanès, J.-L. 2012. "La Chronique du règne de Charles IX ou le "gai savoir" ". Cahiers Mérimée 4: 9-33.

Carbonnier, Y. 1998. "Les maisons des ponts parisiens à la fin du XVIII ${ }^{\mathrm{e}}$ siècle: étude d'un phénomène architectural et urbain particulier». Histoire, économie et société 17 (4): 711-723.

Déruelle, A. 2010. «Histoire de rire: La Chronique du règne de Charles IX». Cahiers Mérimée 2: 41-55.

D'estienne d'Orves, N. 2015. Dictionnaire amoureux de Paris. Paris: Plon.

Dumas, A. 1994. La Reine Margot. Édition établie par Jacques Bony. Paris: Flammarion. [1845].

Durand-Le Guern, I. 2008. Le Roman historique. Paris: Armand Colin. 
Hugo, V. 1907. «Quentin Durward, par Walter Scott». Dans La Muse française 1823-1824. Paris: Édouard Cornély et cie: 25-72. [1823].

Lamartine, A. 1823. Méditations poétiques. Paris: Charles Gosselin. [1820].

Lukacs, G. 2000. Le Roman historique. Traduit par R. Sailley. Paris: Payot et Rivages. [1937].

Magnin, C. 1829. "1572. Chronique du temps de Charles IX; par l'auteur du Théâtre de Clara Gazul». Le Globe 7 (43) : 340-342.

Mathieu-Castellani, G. 2004. «Mérimée et la Corse». Littératures 51: 95-116.

Mérimée, P. 1969. Chronique du règne de Charles IX. Édition préparée et annotée par P. Josserand. Paris: Gallimard. [1829].

Mérimée, P. 1978. Théâtre de Clara Gazul. Romans et Nouvelles. Édition préparée par J. Mallion et P. Salomon. Paris: Gallimard (Bibliothèque de la Pléiade).

Molino, J. 1975. "Qu'est-ce que le roman historique». Revue d'histoire littéraire de la France. 2-3: 195-233.

Mombert, S. 2000. "Le public, le romanesque et l'Histoire. Vigny et Mérimée explorateurs du roman historique». Dans Le Roman historique, sous la direction de D. Peyrache-Leborgne et D. Couégnas. Nantes: Éditions Pleins Feux: 118-133.

Sand, G. 1994. La Rêverie à Paris. Bruxelles: Le Cri. [1867].

Taine, H. 1874. «Prosper Mérimée». Dans Lettres à une inconnue de Mérimée. Paris: Michel Lévy frères: I-XXXV. Vigny, Alfred de. 1980. Cinq-Mars. Édition préparée par A. Picherot. Paris: Gallimard. [1826]. 